\title{
PIERRE DE COUBERTIN Y LOS DERECHOS HUMANOS: DEPORTE COMO DERECHO HUMANO
}

\author{
Dikaia Chatziefstathiou \\ Facultad de Psicología y Ciencias Biológicas \\ Universidad Canterbury Christ Church, Reino Unido \\ dikaia.chatziefstathioui@canterbury.ac.uk
}

Fecha de recepción: septiembre 2020

Fecha de aceptación: noviembre 2020

http://doi.org/10.15366/citius2020.13.2.003

\section{Resumen:}

Los Juegos Olímpicos y Paraolímpicos generan grandes expectativas sobre los derechos humanos debido a su reivindicación de promover el bien común a través del deporte. El fundador de los Juegos Olímpicos modernos, Pierre de Coubertin, ha hecho referencia a menudo a las nuevas tendencias del cosmopolitismo y el internacionalismo. Algunas organizaciones del siglo XIX y XX, los llamados «internacionalismos idealistas», utilizaron el deporte como vehículo principal para su «misión civilizadora», entre los que se incluye el Movimiento Olímpico. Y fue en esta época en la que Coubertin anunció su idea de revivir los Juegos Olímpicos como medio para resolver los enfrentamientos internacionales y mantener la paz mundial. Pero al mismo tiempo su Olimpismo ha sido visto como excluyente, elitista y racista. El «deporte olímpico», se asocia predominantemente con las culturas occidentales, y a pesar de la filosofía del «juego limpio» y la promesa del interculturalismo, el camino hacia la inclusión plena y el reconocimiento de los derechos humanos en los Juegos Olímpicos ha sido inconsistente. Las categorizaciones y discriminaciones raciales, que comparten características con el darwinismo social, fueron parte del Olimpismo operante en los principios de siglo, a pesar de los ideales de igualdad racial y fraternidad. A lo largo de la historia, en el deporte siempre ha habido un debate desafiante sobre el cumplimiento de los derechos humanos. La parte positiva es que los órganos rectores del deporte como el Comité Olímpico Internacional (COI) facilitan la promoción de la paz, la solidaridad y la fraternidad entre las naciones participantes. Sin embargo, debe evitarse una posición evangélica de que el deporte es la solución a todos los problemas.

Palabras clave: deporte, Derechos Humanos, Olimpismo, Pierre de Coubertin

Title: PIERRE DE COUBERTIN AND HUMAN RIGHTS: SPORT AS HUMAN RIGHT

\begin{abstract}
:
The Olympic and Paralympic Games naturally raise high expectations about human rights due to their humanistic claims for promoting the common good through sport. The founder of the modern Olympics, Pierre de Coubertin, has often referred to the new tendencies of cosmopolitanism and internationalism. Some organizations of the $19^{\text {th }}$ and $20^{\text {th }} \mathrm{C}$, the so-called "idealistic internationalism", used sport as a central vehicle for their "civilising mission", including Olympic Movement. And it was in this time that Coubertin announced his idea of reviving the Olympic Games, as means of resolving international confrontations and maintaining world peace. But at the same time his Olympism has been seen as exclusionary, elitist and racist. 'Olympic sport' is predominantly associated with western cultural forms in the form of western codifications. Despite the ideology of 'fair play' and the promise of interculturalism, the way to full inclusion and the recognition of human rights in the Olympics has been uneven. Racial categorisations and discriminations, sharing characteristics with Social Darwinism, were part of the operationalised Olympism of the early century, despite ideals of racial equality and fraternity. Throughout history sport has always been a challenging discourse for the realisation of human rights. The positive side is that sport governing bodies such as the International Olympic Committee (IOC) facilitate the promotion of peace, solidarity and fraternity among the participant nations. However, an evangelical stance that sport is a cure-all in society, however, should be avoided.
\end{abstract}

Keywords: sport, Human Rights, Olympic Movemente, Pierre de Coubertin 
Los Juegos Olímpicos y Paraolímpicos generan grandes expectativas sobre los Derechos Humanos debido a sus reivindicaciones humanistas de promover el bien común a través del deporte. El Comité Olímpico Internacional (COI) afirma que «la práctica del deporte es un derecho humano» (Internacional Olympic Committee, 2016, p.11) y el Comité Paralímpico Internacional (CPI) que «la accesibilidad es un derecho humano básico y un pilar fundamental de justicia social» (International Paralympic Committee, 2015, p.23). Al restablecer los Juegos Olímpicos modernos, su fundador, el barón Pierre de Coubertin, aunque impulsado por una clara agenda política «europea», vio en el deporte un mecanismo alternativo para promover enfrentamientos pacíficos internacionales (Chatziefstathiou, 2012a; 2012b; Chatziefstathiou and Henry, 2007; 2012). Algunos investigadores (Kidd, 1996; MacAloon 1996; Hoberman, 1986, 1995, 2004) afirman que la recuperación de los Juegos Olímpicos por Pierre de Coubertin tenía como objetivo reducir las rivalidades imperialistas de las potencias europeas y la creciente probabilidad de guerra. A finales del siglo XIX el internacionalismo y el cosmopolitismo surgieron como tendencias populares basadas en la cooperación transnacional y global. Los «liberales de Manchester» del siglo XIX Richard Cobden (1804-1865) y John Bright (1881-1889) apoyaron el cosmopolitismo al defender el libre comercio, con la esperanza de que promovería el acuerdo internacional y la interdependencia económica, limitando las posibilidades de una nueva guerra.

$\mathrm{El}$ «cosmopolitismo» se refiere básicamente a la idea de cosmópolis o «estado mundial» y, por tanto, implica la eliminación de las identidades nacionales y la creación de una política común respetada por toda la humanidad. Sin embargo, el término se usa más para describir los esfuerzos hacia la paz y la armonía basadas en los principios de los Derechos Humanos, entendimiento mutuo, tolerancia $\mathrm{y}$, sobre todo, interdependencia (Heywood, 2002). El «internacionalismo» también se refiere al entendimiento internacional, pero está arraigado en supuestos universales sobre la naturaleza humana que la ponen en conflicto con el nacionalismo político (identidad política determinada por la nacionalidad). Puede adoptar la forma de «internacionalismo liberal», que se basa en el individualismo y se refleja en la creencia de que los Derechos Humanos Universales deben trascender la autoridad soberana de la nación. También puede adoptar la forma de «internacionalismo socialista» (o internacionalismo proletario), que se basa en la creencia de la solidaridad internacional y sigue la presunción de una humanidad común (Heywood, 2002). Coubertin ha hecho referencia a menudo a las nuevas tendencias del cosmopolitismo y el internacionalismo, vistos como resultado de las condiciones modernas (especialmente de la rapidez de la comunicación). Con respecto al cosmopolitismo, argumentó que «estaba en aumento en todos los ámbitos». La velocidad comenzaba a tener sus efectos negativos y la gente ya repetía esa expresión inteligente y estúpida «el tiempo es dinero» (Coubertin, 1929, p. 572). En relación con el internacionalismo, desarrolló en varias obras su propia comprensión del concepto, destacando siempre que no contradecía ni socavaba la soberanía nacional.

«Señores, hay dos tendencias en los deportistas modernos sobre las que me gustaría llamar su atención. Se está volviendo democrático e internacional. Su revolución social que ahora se ha logrado entre los hombres* y que también podría lograrse en términos de cosas* como bien explica la primera de estas características; la velocidad del transporte, y la frecuencia de las comunicaciones explican el segundo»(Coubertin, 1894, p. 537).

Una agenda similar a la del cosmopolitismo fue compartida por otros «internacionalismos idealistas» de los siglos XIX y XX que utilizaron el deporte como vehículo principal para su «misión civilizadora». Algunas de las organizaciones más conocidas de este periodo, los llamados «internacionalismos idealistas», incluyen al Comité Internacional de la Cruz Roja (1863), al Esperanto (1887), el Movimiento Olímpico (1894) y el Movimiento Scout (1907) (Hoberman, 1995, 2004). Todas estas organizaciones lidiaron con las ansiedades sobre la guerra y la paz, y difundieron promesas de emancipación o liberación de las rivalidades internacionales de este periodo. La rapidez de la comunicación, la complejidad del comercio moderno y las interdependencias que se crearon, estimularon la confianza y el optimismo de las personas sobre la armonía internacional. El optimismo de la época se evidencia en el discurso del embajador 
británico en Estados Unidos en un banquete celebrado en 1868 en honor al inventor del telégrafo, Samuel Morse, «el cable telegráfico, el nervio de la vida internacional, que transmite el conocimiento de los acontecimientos, elimina las causas de los malentendidos, y promueve la paz y la armonía en todo el mundo» (Standage, 1999, citado por Hoberman, 2004, p. 179).

En esta «época de optimismo», Coubertin anunció su idea de revivir los Juegos Olímpicos como medio para resolver los enfrentamientos internacionales y mantener la paz mundial. El discurso de Coubertin hablaba de ideales universales y de igualdad de oportunidades para todas las personas y todas las naciones. Pero al mismo tiempo su Olimpismo ha sido visto como excluyente, elitista y racista. A pesar de estas aspiraciones de universalismo, el producto cultural del COI «deporte olímpico», se asocia predominantemente con las culturas occidentales en forma de reglamentaciones occidentales del deporte y los juegos, y sus miembros y las principales fuentes de financiación están dominadas por personas e intereses occidentales. La noción de amateurismo, un concepto desarrollado por las clase media y alta victorianas para legitimar la exclusión de las clases trabajadora del deporte, es incompatible con el llamado Olimpismo del «deporte para todos», que se inició y desarrollo principalmente después de la Primera Guerra Mundial. A pesar de la filosofía del «juego limpio» y la promesa del interculturalismo, el camino hacia la inclusión plena y el reconocimiento de los derechos humanos en los Juegos Olímpicos ha sido inconsistente. La exclusión de las mujeres y la provisión de un «podio para la celebración de competiciones, físicas masculinas en contraposición a otras masculinidades y a la feminidad» (Wamsley 2004, p. 235) también ha planteado preguntas sobre los ideales «universales» del Olimpismo durante los primeros años del movimiento. En este contexto Chatziefstathiou and Henry (2012) han demostrado que Coubertin y sus compañeros fueron presionados para permitir la inclusión de las mujeres y las clases trabajadoras.

Además, las estructuras sociales del imperialismo que definieron las relaciones coloniales y produjeron imágenes poderosas de los «Otros» también se reflejan en la expresión del Olimpismo durante los primeros años de su expansión por Europa (1918-1937). En este periodo, las ideologías de la «raza» y de la supremacía blanca surgieron en el pensamiento europeo, lo que promovió un modelo de humanismo occidental etnocéntrico (Davies, 1997). Durante este periodo, se representaron muchas exposiciones orientadas al imperialismo, donde grupos «raciales» y étnicos no europeos, principalmente africanos y asiáticos, mostraron evidencias de su proceso de «modernización» en exhibiciones culturales. El Olimpismo, ideológicamente vinculado con el humanismo europea racialmente excluyente de la época, se distorsionó en la práctica hacia los intereses de las naciones e imperios occidentales «modernos» y «avanzados» (Chatziefstathiou, 2007). Las categorizaciones y discriminaciones raciales, que comparten características con el darwinismo social, fueron parte del Olimpismo operante en los principios de siglo, a pesar de los ideales de igualdad racial y fraternidad. Coubertin, como se demuestra en sus obras, ha atribuido con frecuencia características raciales a los pueblos indígenas de Asia («flexibilidad») (Coubertin, 1931) y de África («perezoso») (Coubertin, 1997), mientras que, por ejemplo, percibía que la «raza blanca» era la principal fuerza civilizadora del mundo (Coubertin, 1908). Todas estas contradicciones han llevado a John Hoberman (1986, p.39) a caracterizar al Movimiento Olímpico como una práctica de «universalismo amoral».

Kidd (2020) afirma que, mientras que los Juegos Originales de Coubertin se caracterizaron por prejuicios patriarcales, imperiales y aristocráticos, la lógica del internacionalismo pacífico que predicaba ayudó a los excluidos a perseguir ideas similares. Esto se muestra en los ejemplos de movimientos de resistencia como los Juegos Olímpicos de Mujeres liderados por Alice Milliat y los Juegos Olímpicos de los Trabajadores impulsados por los partidos socialistas y los sindicatos. Y, posteriormente, desde la década de los 1940 hasta la de 1990 particularmente en África, el deporte en términos generales fue fundamental en las luchas por la resistencia de poblaciones colonizadas (Baker and Mangan, 1987) y en el movimiento antiapartheid, impulsado inicialmente por estados africanos independientes (Booth 1998). Curiosamente, el rol del deporte en la lucha contra la violación de los derechos humanos ha captado la imaginación popular y destacado en películas de Hollywood (por ejemplo, la película 
Invictus dirigida por Clint Eastwood y protagonizada por Morgan Freeman como Nelson Mandela).

El deporte moderno ha sido conocido por sus usos y manifestaciones hegemónicas y contrahegemónicas. Las ideologías dominantes de clase, género y raza se han fortalecido, pero también disputado ampliamente a través del deporte (por ejemplo, las tensiones coloniales y poscoloniales en el rugby y el cricket en África y Asia; la separación de la unión de rugby «amateur» y la liga de rugby «profesional» en Inglaterra; los Juegos Olímpicos de Mujeres y Trabajadores a finales de la década de 1920 en oposición a los Juegos Olímpicos sexistas y clasistas, etc.). El deporte ha sido utilizado como una herramienta político-ideológica y sociocultural muy importante en la configuración de las relaciones globales y siempre ha jugado un papel clave en la globalización, las acciones y actividades de las Organizaciones No Gubernamentales (ONGs), la negociación y reconciliación nacional, el desarrollo del estadonación, las relaciones de poder, ayuda y desarrollo, las organizaciones transnacionales y la alineación, entre otros. Gillian Tett comentó en su artículo en el Financial Times:

«Es en torno al tema de los deportes donde las cuestiones enredadas del poder, el dinero, el racismo y la cultura cristalizan con particular claridad, tanto que algunos antropólogos modernos piensan que los deportes son en realidad muchos más interesantes que la religión en el mundo occidental actual, en lo que respecta a su poder cultural y económico» (Tett, 2014).

A lo largo de la historia, en el deporte siempre ha habido un debate desafiante sobre el cumplimiento de los Derechos Humanos. Desde finales del siglo XVIII hasta mediados del siglo $\mathrm{XX}$, los colonizadores europeos utilizaron el deporte (principalmente el cricket y el fútbol) como un medio de control imperial de los pueblos indígenas en territorios fuera de Europa. Por otro lado, en las luchas étnico-nacionales el deporte ha desempeñado un papel catalizador en la intensificación de los conflictos (por ejemplo, la «guerra del fútbol» de América Central o en los Balcanes o en Irlanda del Norte). A menudo, el deporte se ha relacionado con expresiones de chovinismo, militarismo y nacionalismo violento.

Pero la parte positiva es que los órganos rectores del deporte como el Comité Olímpico Internacional (COI) facilitan la promoción de la paz, la solidaridad y la fraternidad entre las naciones participantes. Como se mencionó anteriormente, uno de los mayores estímulos para el renacimiento de los Juegos Olímpicos fue la aspiración de Coubertin de fomentar solidaridad entre las naciones del mundo a través de enfrentamientos deportivos pacíficos. El COI a lo largo de su historia se ha comprometido activamente con la política de los derechos humanos. Como se mencionó anteriormente proclaman en su Carta «el deporte es un derecho humano» (Chatziefstathiou, 2012a). Colaboran estrechamente con organismos transnacionales (por ejemplo, la ONU) y ONG internaciones (como «Rigth to Play») cuyo objetivo es «poner el deporte al servicio del desarrollo armonioso del hombre (sic), con vista a promover una sociedad pacífica preocupada por la protección de la dignidad humana» (International Olympic Committee, 2016, p.10).

Más recientemente, desde mediados de la década de 1990 en adelante, se fundaron varias instituciones transnacionales de Sport for Development and Peace (SDP) como centros globales para el desarrollo de la paz utilizando el deporte como «lenguaje universal» (por ejemplo, la plataforma Sport and Development en Suiza, Peace and Sport en Mónaco, Right top lay en Toronto y Streetfootballworld en Berlín). La Organización Mundial de las Naciones Unidas $(\mathrm{ONU})$ ha reconocido desde hace mucho tiempo la contribución del deporte al desarrollo y la paz, y la colaboración entre el COI y la ONU ha desempeñado un papel fundamental en la difusión de la aceptación del deporte como medio para promover los objetivos de desarrollo acordados internacionalmente (IOC, 2020). En 2015, se vivió un momento histórico para deporte y el Movimiento Olímpico, el deporte fue reconocido oficialmente como «promotor importante» del desarrollo sostenible y se incluyó en la Agenda 2030 de la ONU (IOC, 2020). 
En los últimos años, algunos hitos históricos fortalecieron la asociación entre el COI y la ONU, que se remontan a 1922, cuando el COI y la Organización Internacional del Trabajo (ILO) establecieron una cooperación internacional.

- En 2009, la Asamblea General de la ONU concedió el estatus de Observador Permanente. Esta decisión permite al COI estar directamente involucrado en la Agenda de la ONU y asistir a reuniones de la Asamblea General de la ONU, donde puede intervenir, teniendo de esta manera la oportunidad de promover el deporte en un nuevo nivel.

- En abril de 2014, la ONU y el COI firmaron un acuerdo destinado a fortalecer la colaboración entre las dos organizaciones al más alto nivel. El acuerdo destacaba que el COI y la ONU «comparten los mimos valores de contribuir a un mundo mejor y pacífico a través del deporte».

- También se anunció entonces que el presidente honorario del COI, Jaques Rogge, había sido designado como Enviado Especial del Secretario General de la ONU para los Jóvenes Refugiados y el Deporte.

- En noviembre de 2014, la ONU reconoció la autonomía del COI y del deporte. La resolución de la ONU reconoce «el deporte como un medio para promover la educación la salud, el desarrollo y la paz», y destaca la importancia del papel del COI y el Movimiento Olímpico en el logro de estos objetivos.

- En diciembre de 2016, las Naciones Unidas elogiaron una vez más al deporte por su poder para estimular el cambio social.

Además, en 1993, la Asamblea General de la ONU aprobó una resolución que consolidó aún más la cooperación COI-ONU con la decisión de recuperar la tregua olímpica, aprobando una resolución titulada «Construir un mundo mejor y pacífico a través del deporte y el ideal olímpico» que insta a los Estados miembros, antes de cada edición de los Juegos, a respetar la tregua olímpica y a cooperar con el COI y el Comité Paraolímpico Internacional «en sus esfuerzos por hacer del deporte una herramienta para promover la paz, el diálogo y la reconciliación en las zonas de conflicto durante y más allá del periodo de los Juegos Olímpicos y Paralímpicos» (IOC; 2020). Sin embargo, algunos ejemplos como Berlín 1936 («Los Juegos Olímpicos Nazis»), Pekín 2008 (la campaña de Amnistía Internacional por los escasos antecedentes de derechos humanos en China) y Sochi 2014 (la Asociación Internacional de Lesbianas y Gays por el historial de derechos de los homosexuales en Rusia) ha demostrado que el deporte es un escenario desafiante para realizar afirmaciones tales como «promoción de la paz», «cosmopolitismo» y «ciudadanía global» a través del deporte. De manera similar, el «ideal» de «Tregua Olímpica» a menudo ha sido desvirtuado y cuestionado como, por ejemplo, en el caso de los conflictos entre Ucrania y Rusia durante los Juegos de Sochi.

Un enfoque asimilacionista (como es por ejemplo a nivel nacional en el republicanismo laico francés) en el cual los ciudadanos de un estado nacional o, en el caso de los Juegos Olímpicos, los miembros de los organismos o movimientos multinacionales, acuerdan regirse por normas definidas centralmente; un enfoque multiculturalista (como se ha asociado tradicionalmente a nivel nacional con, por ejemplo, la política en Gran Bretaña) en el que todas las culturas y civilizaciones se las considera de igual valor y se las debe tratar con igualdad; $y$ un enfoque interculturalista en el que los «mejores» aspectos de varias culturas quedan inmersas en una visión más amplia y compartida del mundo.

Un ejemplo de un evento deportivo reciente se relaciona con la FIFA, que prohibió el uso del hiyab en los primeros Juegos Olímpicos de la Juventud en 2010, lo que efectivamente habría excluido al equipo de fútbol femenino iraní. Esto se hizo por dos motivos, primero, por seguridad (un jugador podría ser ahogado), y segundo, la regla de que no se permitiría en el campo de juego 
ropa que signifique una vinculación religiosa. Después de un período de discusión relativamente intensa entre la FIFA, el COI y el CON iraní, se llegó a un acuerdo con el cual las niñas usarían una gorra especialmente diseñada para ellas. Sin embargo, cuando Irán debía jugar contra Jordania en un partido de clasificación para los Juegos de 2012, las jugadoras aparecieron en el campo con los hiyabs que cubrían el cuello y las autoridades abandonaron el partido. Irónicamente tres jugadoras del equipo de Jordania se quedaron no participaron en el partido porque también usaban el hiyab (Dorsey, 2011).

Posteriormente, la FIFA levantó la prohibición y autorizó el uso de velo por motivos religiosos durante los partidos. «Es una autorización mundial», dijo el secretario general de la FIFA, Jerome Valcke, quien no negó que la organización de la Copa del Mundo Femenina Sub17 en 2016 por el reino árabe de Jordania jugó un papel importante en dicha autorización. Las diferentes posiciones tomadas sobre el simbolismo religioso en la vestimenta se observan claramente al comparar cuáles han sido las actitudes tradicionales británicas y francesas hacia el uso del hiyab por las mujeres musulmanas y el turbante por los hombres sij (Amara, 2012). El Tribunal Europeo de Derechos Humanos en 2009 ratificó la prohibición de los turbantes en Francia para quienes se encuentren en instituciones públicas como escuelas o la administración pública (Singh, 2009), una situación que contrasta drásticamente con la posición tradicional del Reino Unido en el que los sijs pueden usar turbante no solo en los trabajos comunes, sino también en la policía y las fuerzas armadas, y en los que el uso de turbantes permite una exención de requisito legal de usar el casco de motocicleta.

Los ejemplos mencionados anteriormente, aunque no son exhaustivos, muestran como el deporte, y el deporte olímpico en particular, ha sido un vehículo de cambios político, ideológicos y socioculturales en la sociedad global desde los primeros años del barón Pierre de Coubertin. No cabe duda de que el movimiento olímpico puede contribuir al cumplimiento de los derechos humanos. El discurso olímpico, aunque no es conocido por la persona de a pie, tiene un fuerte carácter normativo que se representa en la ideología humanista del Olimpismo y en los valores olímpicos (Chatziefstathiou and Henry, 2012). Sin embargo, debe evitarse una posición evangélica de que el deporte es la solución a todos los problemas. En cambio, las complejidades involucradas en el cumplimiento de los derechos humanos serán reconocidos especialmente por el COI y el IPC, quienes deben ser pragmáticos y proactivos dados sus poderes y limitaciones.

\section{Referencias bibliográficas}

- Amara, M. (2012). Veiled Women Athletes in the 2008 Beijing Olympics: Media Accounts, The International Journal of the History of Sport, 29(4). https://doi.org/10.1080/09523367.2012.658194 (accessed 18 June 2020).

— Baker, W., \& Mangan, J.A. (1987) (eds) Sport in Africa, London: Africana Publishing

— Booth, D. (1998) The Race Game: Sport and Politics in South Africa, London: Frank Cass.

- Chatziefstathiou, D. (2006). All sports for all people": the socialist challenge, Coubertin and the ideology of "popular". Olympism. Kinesiologia Slovenica, 12(2), 13-22

- Chatziefstathiou, D. (2007). The History of Marketing an Idea: The Example of Baron Pierre de Coubertin as a Social Marketer, European Sport Management Quarterly (EASMQ),7(1), 55-80, March 2007. https://doi.org/10.1080/16184740701270337

- Chatziefstathiou, D. \& Henry, I.P. (2007) Hellenism and Olympism: Pierre de Coubertin and the Greek challenge to the early Olympic movement, Sport in History, 27(1), pp. 24-43. https://doi.org/10.1080/17460260701231034 
- Chatziefstathiou, D., \& Henry, I. P. (2012). Discourses of Olympism: From the Sorbonne 1894 to London 2012. London: Palgrave Macmillan. https://doi.org/10.1057/9781137035561

- Chatziefstathiou, D. (2012a). Active Citizens and Public Policy: The Example of London 2012 Olympic Games. International Journal of Sport Management, Recreation and Tourism, 9, 23-33. https://doi.org/10.5199/ijsmart-1791-874X-9b

- Chatziefstathiou, D. (2012b). Olympic Education and Beyond: Olympism and Value Legacies from the Olympic and Paralympic Games. Educational Review, 64, (3), 385-400, 1 August 2012. https://doi.org/10.1080/00131911.2012.696094

- Coubertin, P. (1894). The Neo-Olympism. Appeal to the People of Athens (November 16, 1894). Lecture given to the Parnassus Literary Society at Athens. In N. Müller (Ed.), Pierre de Coubertin 1863-1937: Olympism: selected writings, (pp. 533-254). Lausanne: International Olympic Committee.

- Coubertin, P. (1929). Olympia. lecture given in Paris, in the Festival Hall of the 19th Arrondissement Town Hall. In N. Müller (Ed.), Pierre de Coubertin 1863-1937: Olympism: selected writings, (pp. 563-576). Lausanne: International Olympic Committee.

- Coubertin, P. (1931). Olympic Memoirs. Lausanne: International Olympic Committee.

- Coubertin, P. (1997). The Capitol in Rome (1923). In N. Müller (Eds.), Pierre de Coubertin 1863-1937 - Olympism: Selected Writings, (pp. 495-499). Lausanne: International Olympic Committee.

- Coubertin, P. (1908). Why I Revived The Olympic Games. In N. Müller (Ed.), Pierre de Coubertin 1863-1937 - Olympism: Selected Writings, (pp. 542-546). Lausanne: International Olympic Committee,

— Davies, T. (1997). Humanism. London: Routledge.

- Dorsey, J. (2011). FIFA bans Jordanian women soccer players for wearing the hijab. Al Arabiya News. Recuperado de https://www.alarabiya.net/articles/2011\%2F06\%2F17\%2F153670.html

— Heywood, A. (2002). Politics. London: Palgrave.

- Hoberman, J. 1986. The Olympic crisis: Sports, politics and the moral order., New Rochelle, NY: Aristide D. Caratzas.

- Hoberman, J. (1995). Toward a theory of Olympic internationalism. Journal of Sport History, 22(1), 1-37.

- Hoberman, J. (2004). Sportive nationalism and globalization. In J. Bale and M. K. Christensen (Eds), Post-Olympism? Questioning sport in the twenty-first century (pp. 177-188). Oxford: Berg. https://doi.org/10.4324/9781003086437-12

- International Olympic Committee (2020). Cooperation with the UN. https://www.olympic.org/cooperation-with-the-un (accessed 18 June 2020)

— International Olympic Committee (2016). The Olympic Charter. Lausanne: IOC.

— International Paralympic Committee (2015). IPC Accessibility Guide. Bonn, IPC. 
- Kidd, B. (2020) (in press). The Olympic and Paralympic Games and Human Rights. In D. Chatziefstathiou, B. Garcia and B. Seguin (Eds), Routledge Handbook of the Olympic and Paralympic Games. Routledge: London. https://doi.org/10.4324/9780429440311-34

- Kidd, B. (1996). Taking the rhetoric seriously: proposals for Olympic education. Quest, 48, 82-92. https://doi.org/10.1080/00336297.1996.10484180

- Kotler, P., \& Roberto, E. L. (1989). Social marketing strategies for changing public behaviour. New York: The Free Press.

- MacAloon, J. (1981). This great symbol: Pierre De Coubertin and the origins of the Modern Olympic Games. Chicago, IL: University of Chicago Press.

- MacAloon, J. (1996). Humanism as political necessity? Reflections on the pathos of anthropological science in Olympic contexts. Quest, 48, 67-81. https://doi.org/10.1080/00336297.1996.10484179

- Singh, T. (2009). European Court rules against the Sikh turban in French schools. Sikh Business World.com. http://www.sikhbusinessworld.com/f59/european-court-rules-againstsikh-turban-58655/ (accessed 21 October 2011)

- Tett, G. (2014), 'Notes on a College Scandar', Financial Times, 16 April, https://www.ft.com/content/0714e580-c042-11e3-bfbc-00144feabdc0 (accessed 18 June 2020)

- Wamsley, K.B., (2004). Laying Olympism to Rest. In: J. Bale and M.K. Christensen, eds, Post-Olympism? Questioning Sport in the Twenty-first Century. Oxford: Berg, pp. 231-242. https://doi.org/10.4324/9781003086437-16 